\title{
К. ЯАНИМЯГИ
}

\section{ПРОЦЕДУРА ВЫБОРА МЕРОПРИЯТИЙ ПО ЭКОНОМИИ ТОПЛИВНО-ЭНЕРГЕТИЧЕСКИХ РЕСУРСОВ}

\author{
K. JAANIMĂGI. KOTUSE- JA ENERGIARESSURSSIDE KOKKUHOIU ORITUSTE \\ VALIKU PROTSEDUUR \\ K. JAANIMAGI. THE CHOICE PROCEDURE OF THE ECONOMY ACTIONS OF \\ THE FUEL-ENERGY RESOURCES
}

\section{(Представил Н. Эпик)}

В последние годы широкое развитие получили методы принятия решений в ситуациях выбора. Разумное применение этих методов позволяет раскрыть перед лицом, принимающим решение (ЛПР), суть выбора, сознательно разработать компромиссы, более последовательно проводить в жизнь определенную политику $\left[{ }^{1}\right]$. Применение таких методов для решения сложных задач выбора позволяет существенно расширить возможности ЛПР, усилить его интеллект. В настоящей статье рассмотрена задача выбора совокупности мероприятий по экономии топливно-энергетических ресурсов в условиях ограниченности капитальных вложений на экономию.

Предлагаемая человеко-машинная процедура для решения этой задачи базируется на использовании методов многокритериального анализа, при этом функционалы и ограничения приводятся в виде понятных для ЛПР показателей с учетом требований, предъявляемых к таким процедурам, сформулированным в $\left[{ }^{2}\right]$.

При распределении меропрятий по экономии при остром дефиците капитальных вложений необходимо дифференцировать экономию энергоресурсов в зависимости от их стоимости, вида энергии $\left[{ }^{3}\right]$ (нефть, уголь, пиковая или провальная электроэнергия), рассматривая только те мероприятия, которые обеспечивают бо́льший эффект по сравнению с затратами на развитие топливно-энергетического комплекса страны и на получение дополнительного количества топлива (это условие либо вводится в ограничение предлагаемой модели, используя стоимость или замыкающие затраты, либо только рассматриваются соответствующие мероприятия).

Модель имеет вид:

$$
\begin{aligned}
& f_{1 j}=\sum_{i \in I} \exists_{i 1 j} x_{i} \rightarrow \max , \\
& f_{2}=\sum_{i \in I} \ni_{i 2} x_{i} \rightarrow \max , \\
& f_{3}=\sum_{i \in I} \exists_{i 3} x \rightarrow \max , \\
& f_{4}=\sum_{i \in I} \mathrm{U}_{i} x_{i} \rightarrow \max , \\
& f_{5}=\sum_{i \in I} \Delta p_{i} x_{i} \rightarrow \max , \\
& \sum_{i \in I} \mathrm{~K}_{i} x_{i}=\mathrm{K},
\end{aligned}
$$




$$
1 \geqslant x_{i} \geqslant 0,
$$

где $j$ - вид топлива, $i$ - номер мероприятия по экономии, $l=1,2,3-$ вид энергии, $\ni_{i 1 j}, \vartheta_{i 2}, \ni_{i 3}-$ экономия $j$-го топлива, электроэнергии и тепловой энергии в результате осуществления $i$-го мероприятия, $\mathrm{K}_{i}-$ капитальные вложения на осуществление $i$-го мероприятия по экономии топливно-энергетических ресурсов, $x_{i}$ - интенсивность осушествления $i$-го мероприятия по экономии, $I$ - обшая совокупность мероприятий, $\mathrm{K}$ - суммарный лимит капитальных вложений, $Ч_{i}-$ трудовые затраты при осуществлении $i$-го мероприятия, $\Delta p_{i}$ - прирост производительности труда от внедрения новой технологии (руб.).

Так как вид электроэнергии (пиковая или провальная) и некоторые другие факторы (внеэнергетический эффект) формально из-за отсутствия полного набора исходных данных учесть в модели трудно, то для решения исходной задачи предлагается использовать человеко-машинную процедуру. При этом в качестве активной стороны выступает руководитель (ЛПР) или совет программы по экономии энергии. Процедура решения залачи распределения мероприятий носит итерашионный характер. Следует отметить, что в силу разноразмерности шелевых функционалов их шелесообразно предварительно свести к безразмерным величинам [ $\left.{ }^{4}\right]$.

На первом шаге рассматриваемой прошедуры ЛПРv ппедлагается вектор ндеальных решений, т. е. вектор значений $f_{m}$ опт $=f_{m}\left(x_{i}\right.$ опт, $\left.m\right)$, где $\left\{x_{i}^{\text {oпт, } m\}_{m=1.5}}-\right.$ решение задачи $(m),(6)-(7)$, где $m=1,2,3,4,5$. Совершенно очевидно, что в области (6)-(7) не сvшествvет такого $\left\{x_{i}\right\}$, чтобы вектор значений функшионалов задачи (1)-(7) в этой точке совпадал с $\overrightarrow{f^{0}}=\left\{f_{m}^{\circ \pi т}\right\}_{. m=1,5}$. На втором шаге методами из $\left[{ }^{5}\right]$ строится область Парето задачи $(6)-(7)$. На третьем шаге ЛПР определяет допустимую образ-шель или вектор $\overrightarrow{f^{1}}=\left\{f_{m}^{1}\right\}_{, m=1.5}$.

При определении $\overrightarrow{f^{1}}$ ЛПр учитывает необходимость выполнения общереспубликанских заданий по экономин энергоресурсов, неформализуемые факторы. ЛПР определяет $\overrightarrow{f^{1}}$ покомпонентно на основе сравнения с $\overrightarrow{f^{0}}$.

На четвертом шаге из области Парето выбирается совокупность решений, обеспечивающих для целевых функционалов (1)-(5) значения не ниже, чем $\vec{f}^{1}$. Если таких решений нет, то на следующем шаге ЛПР корректирует свою шелевую программу, формируя вектор $\overrightarrow{f^{2}}$ (как и на третьем шаге), и на следующем шаге повторяет действия четвептого шага и т. д. В противном случае из совокупности таких решений выбирается решение наименее vдаленное от идеального $\left\lceil^{4,5}\right\rceil$ и принимается за оптимальное компромиссное решение - совокупность мероприятий по экономии энергоресурсов. Отметим также, что если ЛПР не считает возможным ухудшение какой-либо из компонент вектора $\vec{f}$, то он может внести изменения в ограничение задачи (6)-(7), расширив область допустимых значений.

Исходную задачу можно сформулировать и как одноцелевую со стоимостным целевым функшионалом, выражающим затраты на единицу сэкономленной энергин. Однако в этой постановке задача имеет ряд очевидных недіостатков, связанных как с завуалированием вида энергии (мазут или тепловая и т. д.), так и со сложностью формирования единого стоимостного критерия. Кроме того, многоцелевая по- 
становка позволяет удобно осуществлять диалоговый режим, анализировать пути выполнения установленных сверху заданий по экономии топлива и энергии.

Для решения рассмотренной в данной статье задачи на ӘВМ нами составлены программы, которые опробованы на конкретном материале, полученном в секторе общей энергетики Института термофизики и электрофизики АН ӘССР в ходе реализации республиканской программы «Әкономия энергии». Разработанный алгоритм и программа показали хорошую работоспособность для задач средней размерности. Программа написана на языке ФОРТРАН и реализована на ЕС 1052 Института кибернетики АН ЭССР. Из-за отсутствия полного набора данных по трудовым затратам на осуществление мероприятий по экономии энергии $f_{4}$ нами не рассматривался. Алгоритм и программа расчета исходной задачи позволяют дать плановым органам рекомендации по эффективному распределению капитальных вложений между отраслями и предприятиями народного хозяйства в случае их дефицита, причем под эффективным распределением можно понимать и распределение на основе методов решения задачи (1)-(7), рассмотренных в. $\left.{ }^{5}\right]$.

\section{Л И ТЕРА Т Р А}

1. Ларичев О. И. В кн.: Проблемы и методы принятия решений в организационных системах управления. М., Изд. ВНИИ сист. исслед., 1982, 5-12.

2. Ириков В. А. В кн.: Проблемы и методы принятия решений в организационных системах управления. М., Изд. ВНИИ сист. исслед., 1982, 12-24.

3. Изв. АН СССР, Энергетика и транспорт, 32, № 5, 11-115 (1982).

4. Яанимяги К. Изв. АН ЭССР, Физ. Матем., 28, № 4, 362-364 (1979).

5. Вайк Л. Э., Рабкин $Г$. Б., Я̆анимяги K. Э. Согласованная оптимизация топливноэнергетических балансов экономических районов: Теория и методы. Таллин, AH ЭССР, 1982.

Ннститут термофизики и электрофизики Академии наук Эстонской ССР

Поступила в редакцию 9/III 1983 